Poznań Studies in Contemporary Linguistics 45(1), 2009, pp. 43-54

(C) School of English, Adam Mickiewicz University, Poznań, Poland

doi:10.2478/v10010-009-0001-y

\title{
NATURAL PHONOLOGY AS A FUNCTIONAL THEORY
}

\author{
ANNA BALAS \\ Adam Mickiewicz Unviersity, Poznań \\ abalas@ifa.amu.edu.pl
}

\begin{abstract}
This paper presents Natural Phonology as a functional theory. Natural Phonology is shown to be functional in two senses: as focusing on explanation and thus increasing our understanding of how language works, and as having practical applications, especially to second language acquisition and speech therapy. The contribution argues that crucial as formalism is in computational linguistics and speech technology, Natural Phonology, with less rigid and less formalized claims, has important applications in the areas where language and not totally predictable human factors are involved. The paper discusses approaches to autonomy in language, explanation and hypothesis in Natural Phonology, and applications of Natural Phonology.
\end{abstract}

KEYWORDS: Natural Phonology; functionalism; second language acquisition.

\section{Introduction}

A prerequisite for any discussion on the usefulness of a given theoretical approach to language research is agreement on what the goals of research are. Such an agreement seems not to have been reached in phonology between formalists and functionalists who argue about the essence and role of description, explanation and practical applications. This paper discusses aspects of functionalism and formalism in relation to Natural Phonology as a reaction to Gibbon's (2007) paper. Gibbon (2007) implies that Natural Phonology is anti-formalist and claims that in order to be operationally functional Natural Phonology should express itself more formally, more computationally. This contribution argues that although Natural Phonology emphasizes function, it is not anti-formalist. Natural Phonology is presented as a functional theory, where "functional" means both focusing on explanation and offering practical applications. As regards the first meaning, Natural Phonology focuses on explanation, on answering the "why" questions rather than on precise description, as formal theories do. To illustrate the interests of the theory, the following sections discuss approaches to autonomy in language, the nature 
of explanation, and the nature of hypothesis in Natural Phonology. With relation to operational functionality, Section 5 shows where Natural Phonology can be practically applied, answering the needs of the context of a utilitarian ecology of science (cf. Gibbon 2007).

\section{Autonomy}

For the approach favoring direct technological implementations postulated by Gibbon (2007), it seems necessary to assume autonomy of language, because viewing language as a closed, self-contained structure helps in listing and formalizing of the elements involved. Language can, however, be viewed as an autonomous or non-autonomous cognitive faculty. The generativists assume that language is an innate, autonomous faculty, independent of other non-linguistic cognitive abilities. The functionalists are more likely to think that language is a non-autonomous faculty, and they assume that the human ability to use a language is fundamentally not different from other cognitive abilities humans have. Natural Phonology is a functional theory relating language to other domains of human life.

According to Croft and Cruse (2004), the hypothesis that language is not an autonomous cognitive faculty has implications for the representation of linguistic knowledge and processes governing language use. The corollary related to linguistic knowledge is that knowledge of meaning and form on all levels of the language system, i.e. phonology, morphology, syntax and semantics, is conceptual. Although sounds and utterances are physical entities with a formal structure, they must be produced and comprehended. Comprehending and producing speech is possible thanks to cognitive processes which accept speech sounds as input and produce utterances as output. The corollary related to processes governing language is that construction and communication of meaning by language are governed by the same principles as other cognitive abilities. Speaking and comprehending language involves essentially the same cognitive abilities that humans use in visual perception, reasoning or motor control. The component cognitive skills used for language processing are used for other cognitive tasks, too, and only the configuration of cognitive abilities used for comprehending and producing language is unique. This is not to deny an innate human language faculty. What functionalists deny is an autonomous innate human capacity for language. Functionalists acknowledge an innate component that gives rise to linguistic abilities unique to humans, but the emphasis of functionalist research is on the role of general cognitive abilities in language. The hypothesis that language is not an autonomous cognitive capacity has an implication for the functionalist approach to language research in the sense of holistic thinking: an adequate model of language has to involve reference to general conceptual structures and cognitive abilities.

Natural Phonology has always emphasized the role of external factors, such as cognitive abilities and physiological predispositions, in phonological processes. Some 
European natural phonologists replaced the original hypothesis about complete innateness of natural phonological processes by a hypothesis that processes arise at different stages of development as a result of self-organization (Dziubalska-Kołaczyk 1998).

\title{
3. Explanation in Natural Phonology
}

Natural Phonology uses "deductive inferences about grammars based on universal higher-order principles applicable to language as well as to other natural phenomena" (Dziubalska-Kołaczyk 2001: 77). Natural Linguistic preferences are explanatory because they are based on non-linguistic, external evidence.

\begin{abstract}
[W] can arrive at explanations for the regularities within a certain domain by turning to theories that are not theories for that particular domain (e.g., for grammatical theories, these include: theories of phonetic production, perception, learning, memory, communication, action, semiotic theories, etc.).
\end{abstract}

(Vennemann 1983: 9).

Natural Linguistics proposes a "hierarchic, deductive system within which linguistic preferences occupy a general second rank, below higher principles and above the specific linguistic consequences of preferences" (Dressler 1999: 390). DziubalskaKołaczyk (2001: 76) illustrates the levels of the system with the following examples. Higher principles can be cognitive, phonetic, psychological, sociological etc., they are non-linguistic principles, like for example the principle of the least effort. Linguistic preferences include for instance a preference for simple phonotactics, for a CV structure. A linguistic consequence of such a preference is then the absence of clusters in a given language. In a situation of conflict between preferences "agents strive towards maximal benefit or expected utility" (Dressler 1999: 392). Solutions for conflicts between preferences are to be found in higher-order universal principles and not in language-internal properties, because "preferences in the use and acquisition of language become frozen in preferences of language structure" (Dressler 1999: 394). It is also important to note that resolutions of conflicts can be predicted to a certain extent and therefore testable hypotheses can be postulated, but "total predictability is excluded by interlinguistic and intralinguistic language variation" (Dressler 1985: 294-295).

We have to decide whether we want to explain, what we want to explain and how we want to explain. It seems that, for formalists, explanation understood as answering the question "why?" and finding out how the mind works is less important than constructing computational models. According to Gibbon (2007: 83), "[n]either technology nor computational models need be close mirrors of the workings of the mind". He further claims that "[e]xploiting external evidence is not sufficient: theories have full explanatory status only with explicit procedural and computational interpretations within the ecological niche of science itself, and within broader technologies". This approach is not interested in using external evidence to explain how the mind works, 
is not interested in using external evidence to explain how the mind works, but in constructing technological applications.

According to Natural Phonology, "[s]cientific explanation is directed at gaining insight or understanding" and "[t]o ask for a scientific explanation is to ask for one or more reasons why it is as it is and probably cannot be different" (Botha 1981: 188; please note that this quote is taken from an author in no way connected with Natural Phonology). Natural Phonology claims that to describe and to formalize does not mean to explain. Description is a step in scientific endeavors, as the data are needed to verify any hypothesis. Formalization is also useful to test the hypothesis. Explanation, however, is answering the question "why?". Moreover, the answer to this question would not be considered satisfactory if it referred to internal explanation only, i.e. characterized the formal relationships between phonological entities within a self-contained grammar system. Natural Phonology stresses that the function of conveying meaning as well as cognitive and physiological factors influence language to such an extent that it is not feasible to describe and explain language without referring to the broadly understood functions it has.

Unlike many opponents of Natural Phonology seem to imply, it is not the case that Natural Phonology rejects formalism, i.e. very precise statements, e.g. stated in computational terms. Even though not many functionalist phonologists have endeavored to do so, there is nothing in attempting to produce formalized statements that would be incompatible with the Natural Phonological approach. In fact Dziubalska-Kołaczyk's (2002) Beats-and-Binding Phonology is stated in rigid terms. Tobin (this volume) also mentions that Phonology as Human Behavior (Tobin 1995), a theory also interested in answering the question "why?" rather than in formal technology, has been used in a computer program based on lip-reading for the hearing-impaired (Geri et al. 2008). These are examples which show that there is room for technological applications of functional, not formal, phonological theories.

The issue of external evidence and its function in description and explanation has been a matter of debate. According to Natural Phonology, external evidence is vital for explanation. According to its critics, structural explanation would be more useful (e.g. in technological applications), whereas relating preferences to higher-order principles only pushes the problem of explanation one step back. For Natural Phonologists, however, structural description and its formalization are mere descriptions useful before invoking more general, covering laws. The point first raised by Hempel and Oppenheim (1948) and used by Eckman (2008) to defend the Structural Conformity Hypothesis is also useful here. The question "why" can be raised over again and relate to the general laws that serve phonology as explanations. These laws will act as facts for higher-order areas and they themselves can be explained if they can be subsumed under generalizations which are more comprehensive, i.e. if they can be deduced from some more encompassing laws or principles. What is crucial is that Natural Linguistic preferences in the form of law-like statements make testable predictions. 
4. Natural Phonological hypotheses related to acquisition of second language phonology

Natural Phonology, a functional theory, is often used to model and explain first and second language acquisition phenomena and speech therapy issues. This section will show how the nature of Natural Phonology can be useful in research related to predicting acquisition patterns in second language (henceforth L2) phonology.

The choice of Natural Phonology as a framework for L2 speech research is associated with the assumption that phonetic detail plays a crucial role in phonology in L2 acquisition. The suitability of Natural Phonology for L2 acquisition research is seen in its views on the phonetics-phonology relationship, advantages over formal phonological theories, and aims of pronunciation training.

The Natural Phonological hypothesis related to L2 phonology acquisition incorporates reasoning of both phonetic and phonological types, as these two domains of speech are seen as interconnected. Phonetics deals with regularities in speech acoustics typical for a given language, whereas phonology has a twofold task. On the one hand, phonology looks for phonemically meaningful regularities; on the other hand, it tries to explain these regularities and determine why they occur in a given language. In other words, phonology is about the priorities the speech system of a given language has. Phonological reasoning begins as perception in the hearer, with acoustic and physiological conditioning of what is possible in speech. Phonological reasoning then goes up towards higher levels of abstraction, where phonology chooses from what phonetics has to offer on more arbitrary bases. These more arbitrary bases can refer to Natural Phonological system-adequacy whereby the phonology of a language organizes and changes its categories and processes within a system that serves speech production and speech perception. The task of a phonologist is then seen as a search for phonetic details that are crucial in a given language, the word "crucial" referring to phonemic differences and phonetic details responsible for the characteristic of a given language or its accents. The above description of phonology is not intended to be a definition of phonology, but rather as a presentation of a standpoint on the close and complex relationship between phonetics and phonology.

Both in L1 and in L2, hearers/speakers rely on phonemic representations understood as their intentions. Natural Phonological phonemes are both real and mental in that they are the perceived, fully specified sounds, not just lists of abstract features. L1 phonemes form the basis for L2 phoneme formation. Phonological analysis of L2 acquisition has to concentrate on phonetic details differentiating phonemes in L1 and L2 and phonetic details involved in lenitions and fortitions. Reasoning based on the stored phonemic categories only is not capable of accounting for L2 speech phenomena. The stored phonemic categories are insufficient to be used for an analysis of L2 acquisition, because their tertium comparationis has too narrow a spectrum. Haspelmath (2006) emphasized the following: "an important consequence of the non-existence of preestablished categories for language typology is that comparison cannot be category- 
based, but must be substance-based, because substance (unlike categories) is universal". There is a similar issue in L2 acquisition research. Similar as they might seem, phonemes in L1 and L2 do not reflect identical, pre-defined categories, but are specified by each language separately. Each language chooses its own set of sound categories and defines these categories at least slightly differently, specifying phonetic details in a unique way. Therefore no two languages have the same set of sound categories with the same phonetic specifications. Such unique arrangements of sound specifications are possible because, as Dziubalska-Kołaczyk (2004) emphasizes, under universal constraints and language-specific conventions, the phonology of each language chooses from a wide range of options that phonetics offers.

As they do not have access to category specifications according to the phonology of the language they are learning, L2 learners do not know how categories are specified in L2. ${ }^{1}$ At their disposal, correlated with the morphological and syntactic structures they have learned, in terms of L2 speech influence, learners have only the acoustic signal, phonetic detail. In terms of the influence of their first language, they are used to paying attention to some details, but disregarding others. L2 learners do not have access to the phonological system of the L2 other than through phonetics. What reaches their ears is the acoustic signal which has to be deciphered. It is deciphered according to first language processes, and in doubtful cases universal processes apply. The application of L1 and universal processes to L2 acoustic signal leads to the determination of the underlying representations of interlanguage. What is produced by L2 learners is produced on the basis of these, often misperceived, underlying representations, which are in the production direction of speech processing again processed by means of L1 or universal processes. Pronunciation training or mastering consists in learning which phonetic detail to disregard, which L1 processes to suppress, and to which phonetic details attention should be paid. Thus teaching or learning L2 pronunciation consists in teaching or learning what L2 phonology chooses from phonetics.

If phonetic detail is so important in L2 phonology, then a theory chosen to model and explain L2 acquisition should incorporate phonetic evidence into its model. The generative paradigm in phonology (Chomsky 1968) operates on discrete sound segments, and assumes that phonetic segments are formal symbol tokens and that a phonetic space is closed and contains only static symbolic objects (cf. the criticism of formal phonology by Port and Leary 2005). In generative phonology, each segment is

\footnotetext{
${ }^{1}$ A second language teaching method may include pronunciation training and the development of metalinguistic competence in the area of pronunciation. Usually, however, general language courses stress the similarity between the phonemic categories in $\mathrm{L} 1$ and $\mathrm{L} 2$, and overlook the differences in category specification, phonotactic distribution and the application context-sensitive processes. Only those aiming to become language professionals - teachers, interpreters, and linguists - are offered more detailed pronunciation training. Those who study e.g. English for other purposes have to cope with pronunciation problems either by subconscious acquisition or by various compensatory methods. Compensatory methods, like repetition, avoidance, description or the reliance on the interlocutor to decipher the underlying intention, often hinder communication.
} 
composed of simultaneously occurring distinctive features which relate to phonetic features, but only to the extent to which they are needed to express phonological rules. Phonological rules, however, are only responsible for mapping underlying representations onto surface representations. Although it is claimed that a surface, phonetic representation is the form of the word in which it is spoken or heard, in fact the repertoire of distinctive features is too small to account for regional or L2 accent differences, to mention just one of the problems that generative phonology has in modeling speech.

In L2 acquisition, however, the phonemic status of a sound is of secondary importance. What matters in L2 acquisition is phonetic detail as compared with the phonetic details of L1. Therefore generative phonological theories working on phonemic symbols are not able to explain L2 pronunciation, which depends on very fine-grained phonetic detail. Natural Phonology is a theory which sees the acoustic signal, phonetic detail and phonemic categories as belonging to a systematic speech processing continuum, in that phonetic detail is based on what acoustics offers, and phonemic categories are based on what phonetics has to offer, and phonological processes apply already to underlying representations, and shape the sound until it reaches its ultimate phonetic form. Such an ultimate phonetic form is fully specified, and it involves all phonetic details responsible for variations between native accents, or the difference between L1 and L2 speech, etc. Thus Natural Phonology poses no artificial restrictions on L2 acquisition modeling by limiting its apparatus only to the phonemic inventory, and helps incorporate external evidence into phonological explanation.

Predictions can be drawn from Natural Phonology, which offers a relatively limited but powerful set of dynamic, preference-based processes which have so far been used to describe a wide spectrum of phonological phenomena, ranging from first and second language acquisition to aphasia to diachronic changes. Underlying representations, and L1, L2 and universal processes, as understood by Natural Phonology, can be used to model and explain phonological aspects of L2 acquisition.

Adult L2 learners do not start learning L2 in a vacuum. It has long been suggested that L1 acts as a "sieve" filtering out speech features which are not significant in the first language phonological system (Polivanov 1932; Trubetzkoy, 1939/69). L2 learners are equipped with $\mathrm{L} 1$ categories, or to be more precise, underlying representations as specified by L1. A particular contribution of Natural Phonology to L2 phonology research is that dynamic, preference-based, subconscious L1 processes are used to shape sounds and sound sequences in interlanguage production and perception. The use of L2 processes which are not used in L1 may become evident. In new contexts, some universal processes whose use might not be evident in either L1 or L2 are used in L2 acquisition. The use of universal processes, not used in either L1 or L2, is present when the process is covert in L1 because of the lack of a specific context in which it could apply. The use of the process is restricted or suppressed in L2, but L2 learners have not managed to limit the process in accordance with L2 phonology. For example, Japanese learners of English devoice final obstruents although Japanese does not have final obstruents, so it is not a process transferred from L1. With time, more universal and L2 
processes come into play, as the learner notices that $\mathrm{L} 1$ processes are not sufficient to represent L2 sounds.

According to Natural Phonology, the task of the L2 learner consists in deciphering underlying representations in $\mathrm{L} 2$ in the perception direction of speech processing and arriving at phonetic representations by means of processes used in $\mathrm{L} 2$ in the production direction. Vital for native-like pronunciation in L2 is finding out:

- which processes apply in L1 and L2;

- which processes that apply in L1 should be limited in L2;

- which processes which do not play a role in L1 should apply in L2;

- which universal processes which are covert in L1 due to the lack of a specific context are suppressed in L2. Then the learner has to learn to suppress the process in accordance with L2 phonology.

The assumption is that that there are significant regularities to be discovered in the production of L2 speech. These regularities are likely to stem primarily from the use of L1 processes. It is also assumed that there is evidence for the use of processes nonexistent either in L1 or in L2, namely universal processes, which have been latent in L1, because appropriate contexts where they could apply are not present in L1. Also, some processes might be acquired directly from L2 as a result of exposure to L2. A challenging question could be asked why speakers with common L1, exposure to the same L2, and access to universal processes do not speak in exactly the same way in L2; why is there so much phonological variability between what they produce in L2? Even the idea of universal processes is sometimes questioned in light of numerous variants of L2 pronunciation. It is true that phonological variability observed in L2 speakers is typically greater than in the case of L1 adult speakers. It is noteworthy, however, that children acquiring their L1 also display considerable phonological variability. High variability should be simply acknowledged as a property of developing phonological systems. It should not be a priori assumed that the use of a common range of universal and L1 processes (both in the case of L1 and L2 acquisition) and L2 processes (in the case of L2 acquisition) should always yield the same results, as, in order to overcome a given pronunciation difficulty, learners have at their disposal a wide range of processes to use and their choices might vary. The aim is to find common patterns of pronunciation strategies employed by L2 learners, determine their sources and explain post facto their use. The hypothesis about the use of L1, universal, and L2 processes will be defended if it can be shown that L2 learners do not apply processes randomly to overcome pronunciation difficulties. Nevertheless, as in the case of L1, talker variability is also expected. In L2, however, in addition to talker variability, there exists variability stemming from various, though not randomly various, processes employed to overcome pronunciation difficulties. 


\section{Applications of Natural Phonology}

The importance of considering evidence from other domains of human activity, such as cognition, perception, production, memory and learning, as well as providing explanations related to conveying meaning, cognitive and physiological factors is vital in applying Natural Phonology in first and second language acquisition or speech therapy.

The self-organization version of Natural Phonology assumes a constructivist conception of acquisition in which the model of self-organizing processes provides a bridge theory for physiology, psychology, neurology and Natural Phonology. According to this model, phonology results from the interplay of genetic preprograming of phonetic processors and cognitive principles, and input information influencing neuron specialization and development of language modules. In contrast to the strong hypothesis (Donegan and Stampe 1979; Stampe 1969, 1979), the self-organization model predicts that phonological processes are not fully innate, but they "arise at different stages of maturation in alternative set-ups" (Dziubalska-Kołaczyk 1998: 101). Certainly, it would be beneficial if Natural Phonology were able to formalize the stages of self-organization. The formal approach to self-organization of de Boer (2001) focused on proving that selforganization can create vowel systems, and employed too many oversimplifications to be convincingly real: emergent patterns did not convincingly match real systems and there was no connection between the model and actual vowel acquisition or change (Donegan 2004). The following paragraphs show that despite the lack of a rigid formal model, Natural Phonological research can be successfully applied in areas where human factors, less predictable than computer processing, are employed.

Luschützky (1991) prepared an annotated bibliography of naturalism in linguistics. Many authors subscribing to naturalism are listed in Dziubalska-Kołaczyk (2001). Here, three recent studies will be briefly discussed to show how Natural Phonology is applied in second language acquisition and speech therapy.

Połczyńska-Fiszer (2007) examined first and second language dysarthria in traumatic brain injury (henceforth TBI) patients after prolonged coma. On the basis of articulatory processes emerging in child speech in first language acquisition proposed by Natural Phonology and used by Phonology as Human Behavior (Tobin 1997), she developed a taxonomy of characteristic processes found in TBI patients with dysarthria. She showed that the processes in TBI patients were more regular and involved more phonetic motivation more strictly than the processes in first language acquisition. She found several lenition processes which were not previously described in the literature on dysarthria, i.e. incomplete consonant closure and consonant approximation. She demonstrated that on the basis of linguistic analysis of articulatory patches, dysarthria can be diagnosed, and on the basis of knowledge which articulators were weakened, it can be predicted which brain nerves were injured.

Wrembel (2005), using the framework of the Natural Phonological theory of second language acquisition involving self-organization of processes, showed that formal instruction and metacompetence can help second language learners acquire native-like 
pronunciation in a second language. Being told about phonemes, allophones and processes in the two languages lets students concentrate on suppressing and unsuppressing appropriate processes in English as a second language. The theoretical embedding of her research in Natural Phonology, with its emphasis on phonetic and cognitive factors, allowed Wrembel to draw practical conclusions for L2 pronunciation training.

Bogacka (2007) analyzed phonetic and phonological aspects of second language acquisition on the basis of English diphthong production by Polish learners of English. The results relate directly to Natural Phonology's explanatory potential. The results have been explained using the explanatory tools of Natural Phonology, namely processes, with their inherent dynamics. A hierarchy of processes has been reconstructed on the basis of acoustic phonetics, effort management, communicative force and underexercised motor-articulatory patterns. Context-sensitive processes existent in L1, like nasal vocalization, were transferred to L2. Context-sensitive processes from L1 appeared in L2 also when L2 provided a seemingly new context characterized by features which motivate a given process in L1. Such was the case with nasal vocaliztion after diphthongs and before fricatives. Depending on how glottal stop insertion is interpreted, it can be assumed that the preference for CV sequences is strong, or that glottal stop insertion is active when L2 learners need to cope with reading a text in L2. Processes responsible for rhythmic properties and fortitions specifying vocalic quality seem to be relatively easier to acquire than context-sensitive processes. Similar research concentrating on other sounds would allow for constructing a syllabus dedicated to the needs of Polish learners of English mastering English pronunciation.

These are the areas where Natural Phonological findings can be put into practice. Such contexts should be considered the environments where evidence from language use which Natural Phonology takes, is put back and applied as a product, thus closing the ecological circle (cf. Gibbon 2007). This has been the case with Połczyńska-Fiszer (2007), Wrembel (2005) and Bogacka (2007) above, and many other works listed in Luschützky (1991) and Dziubalska-Kołaczyk (2001).

Computational formalisms produce applicable output of phonological modeling. Computers with their speech synthesis or speech recognition systems are, however, supposed to imitate the same results that language users achieve either in speech production or in speech perception. The methods that are used in speech technology are simply expected to be efficient for its purposes, and hence the use of statistical tools or formalisms involving closed data sets, extremely fast processing time and memory storage based in arbitrary ways on features such as alphabetical or numerical order. The methods that are used by humans to achieve the goal, as for example contentaddressable memory with storage by meaning and sound, are not at all important in speech technology as long as the system is efficient. Linguistics, however, is the study of language as used by humans, not the study of speech technology. Therefore, it is postulated that since speech technology is interested in efficiency of computer systems synthesizing or recognizing speech, formalisms are indispensable in its realm, but in the area of Natural Phonology, interested in language as a human phenomenon in the wide 
context, functional considerations and explanatory approach using external evidence, although perhaps not rigidly formalized, are much more useful in the ecological context of speech learning in first and second language acquisition and speech therapy.

\section{Conclusions}

Functionalism of Natural Phonology consists in the theory's focus on explanation, and practical applications. It has been argued in this paper that the basis for a discussion of usefulness of any theoretical approach is agreement on the goals of research. It has been shown that the methods of the study of language and the approach taken, i.e. formalist or functionalist, determine what goals we can aim at. Formalized approaches to language, producing operational computational models, are useful for applications in technological domains, as for instance speech recognition or speech synthesis. Applicable to technology as functional, explanatory approaches sometimes prove to be, they are more focused on language as used by humans. This interest makes them well-suited for applications where the "human factor", with its psychological and physiological embedding, is crucial, as for example in first and second language acquisition or speech therapy. In these domains implicational hierarchies of processes turn out to be successfully applied in teaching children who have difficulties in production in their native language or students striving to make progress in pronunciation in the second language, and in treating patients with aphasia or dysarthria.

\section{REFERENCES}

Bogacka [Balas], Anna. 2007. Repopulating vowel space: English diphthong production by Polish learners of English. (Unpublished PhD dissertation, Adam Mickiewicz University, Poznań.)

Botha, R. 1981. The conduct of linguistic inquiry. The Hague: The Mouton Publishers.

Chomsky, N. and M. Halle. 1968. The sound pattern of English. New York: Harper and Row. Clements, G.N. 2003. "Feature economy in sound systems". Phonology 20. 287-333.

Croft, W. and A. Cruse. 2004. Cognitive linguistics. Cambridge: Cambridge University Press. de Boer, B. 2001. The origins of vowel systems. Oxford: Oxford University Press.

Donegan, P. 2004. "Review of Bart de Boer: The origins of vowel systems". Journal of the International Phonetic Association 34(1). 95-100.

Donegan, P. and D. Stampe. 1979. "The study of Natural Phonology". In: Dinnsen, D.A. (ed.), Current approaches to phonological theory. Bloomington: Indiana University Press. 126173.

Dressler, W. 1985. Morphonology. Ann Arbor: Karoma Press.

Dressler, W. 1999. "On a semiotic theory of preferences in language". In: Haley, M. and M. Shapiro (eds.), The Peirce Seminar papers. Essays in semiotic analysis. New York: Berghahn Books. 389-415.

Dressler, W. and A. Karpf. 1995. "The theoretical relevance of pre- and protomorphology in language acquisition”. Yearbook of Morphology 1994. 99-122. 
Dziubalska-Kołaczyk, K. 1998. "Self-organization in early phonology”. In: Puppel, S. (ed.), Scripta manent. Poznań: Motivex. 99-112.

Dziubalska-Kołaczyk, K. 2001. "Phonotactic constraints are preferences". In: DziubalskaKołaczyk, K. (ed.), Constraints and preferences. Berlin: Mouton de Gruyter. 69-100.

Dziubalska-Kołaczyk, K. 2002. Beats-and-Binding Phonology. Frankfurt am Main: Peter Lang.

Dziubalska-Kołaczyk, K. 2002. "Challenges for Natural Linguistics in the twenty first century: A personal view”. University of Hawai ' $i$ Working Papers in Linguistics 23. 15-39.

Dziubalska-Kołaczyk, K. 2004. "Modern Natural Phonology: The theory for the future". Paper presented at the 35th Poznań Linguistics Meeting, Tarnowo Podgórne.

Eckman, F.R. 2008. "Typological markedness and second language phonology”. In: Hansen, E., G. Jette and M.L. Zampini (eds.), Phonology and second language acquisition. Amsterdam: Benjamins. 95-115.

Gibbon. D. 2007. "Formal is natural: Toward and ecological phonology". In: Trouvain, J. and W. J. Barry (eds.), Proceedings of the 16th International Congress of Phonetic Sciences. Saarbrücken: University of Saarbrücken. 83-88. <http://www.icphs2007.de/>

Haspelmath, M. 2006. "Pre-established categories don't exist - Consequences for language typology and description". Paper presented at the Deutsche Gesellschaft für Sprachwissenschaft Annual Conference, Bielefeld.

Hempel, C. and J. Oppenheim. 1948. "Studies in philosophy of science". Philosophy of Science XV. 135-175.

Liljencrants, J. and B. Lindblom. 1972. "Numerical simulation of vowel quality systems: The role of perceptual contrast". Language 48. 839-862.

Luschützky, H.C. 1991. "Twenty years of naturalism in linguistics: A bibliography”. Wiener Linguistische Gazette, Beiheft 10.

Polivanov, E. 1932. "La perception des sons d'une langue étrangère”. Travaux du cercle linguistique de Prague 4. 79-86.

Połczyńska-Fiszer, M. 2007. First and second language dysarthria in TBI patients after prolonged coma. (Unpublished PhD dissertation, Adam Mickiewicz University, Poznań.)

Port, R. and A. Leary. 2005. “Against formal phonology”. Language 81. 927-964.

Geri, N., S. Neumann, R. Schocken and Y. Tobin. 2008. "An attention economy perspective on the effectiveness of incomplete information". Informing Science 11. 1-15.

Stampe, D. 1969. "The acquisition of phonetic representation". CLS 5. 443-454.

Stampe, D. 1979. Dissertation on Natural Phonology. New York: Garland.

Tobin, Y. 1997. Phonology as human behavior: Theoretical implications and clinical applications. Durham, NC: Duke University Press.

Trubetzkoy, N. 1939/1969. "Grundzüge der Phonologie". Travaux du cercle linguistique de Prague 7.

Vennemann, T. 1983. "Causality in language change: Theories of linguistic preferences as a basis for linguistic explanations". Folia Linguistica Historica 6. 5-26.

Wrembel, M. 2005. "Metacompetence-oriented model of phonological acquisition: implications for teaching and learning second language pronunciation". In: Proceedings of the Phonetics Teaching and Learning Conference. London: University College London. 1-4.

\section{Address correspondence to:}

Anna Balas

School of English, Adam Mickiewicz University

al. Niepodległości 4

61-874 Poznań, Poland

balas@ifa.amu.edu.pl 